\title{
Placental Vascularization and Apoptosis in Rats Orally Exposed to Low Doses of Bisphenol A
}

\author{
Jacopo Troisi ${ }^{1,2 *}$, Luigi Giugliano"${ }^{1 \#, ~ A n t o n i o ~ D ' A n t o n i o ', ~ A n d r e a ~ V i g g i a n o ', ~}$ \\ Rosaria Meccariello ${ }^{3}$, Marika Scafuro', Marcellino Monda', Angelo Colucci', ${ }^{1,2}$ \\ Giovanni Scala², Maria Cofano1, Maurizio Guida',2

\footnotetext{
${ }^{1}$ Department of Medicine, Surgery and Dentistry, "Scuola Medica Salernitana", University of Salerno, Baronissi, Italy

${ }^{2}$ Theoreo srl-Spin-Off Company of the University of Salerno, Baronissi, Italy

${ }^{3}$ Dipartimento di Scienze Motorie e del Benessere, University of Naples Parthenope, Naples, Italy

${ }^{4}$ Department of Experimental Medicine, University of Campania “Vanvitelli”, Naples, Italy

Email:*troisi@theoreosrl.com
}

How to cite this paper: Troisi, J., Giugliano, L., D’Antonio, A., Viggiano, A., Meccariello, R., Scafuro, M., Monda, M., Colucci, A., Scala, G., Cofano, M. and Guida, M. (2018) Placental Vascularization and Apoptosis in Rats Orally Exposed to Low Doses of Bisphenol A. Open Journal of Obstetrics and Gynecology, 8, 958-969.

https://doi.org/10.4236/ojog.2018.811097

Received: July 31, 2018

Accepted: September 1, 2018

Published: September 4, 2018

Copyright $\odot 2018$ by authors and Scientific Research Publishing Inc. This work is licensed under the Creative Commons Attribution International License (CC BY 4.0).

http://creativecommons.org/licenses/by/4.0/ (c) (i) Open Access

\begin{abstract}
Aim: Exposure to the ubiquitous endocrine disrupter Bisphenol A (BPA) has been associated, in pregnancy, with low birth weight. The aim of our study is the identification of the damage caused by Bisphenol A on placental tissue through the evaluation of its effects on micro-vessel density and apoptosis. Methods: After fertilization, we exposed 3 female rats to oral BPA, by means of a free access to a beverage solution containing $100 \mu \mathrm{g} / \mathrm{L}$ of BPA. Three female rats were used as controls. Placentas underwent histological examination and immunohistochemistry for von Willebr and factor (F-VIII) and caspase-9. Results: Sixty-seven fetuses have been produced, 30 from control rats and 37 from exposed rats. Exposed fetuses showed a lower longitudin$\mathrm{al} /$ transverse diameter ratio than controls $(2.57 \pm 0.29$ vs. $2.78 \pm 0.38, \mathrm{p}<$ 0.05). Also, exposed fetuses showed a significant reduction in the number of placental vessels per field $(124.86 \pm 19.15$ vs. $143.54 \pm 22.09, \mathrm{p}<0.05)$. On the other hand, apoptosis is not increased by exposure, as shown by caspase- 9 levels. Conclusion: Exposure to BPA during pregnancy may affect placental vascularization, and this phenomenon may explain the lower birth weight reported. However, our results do not show the increase in apoptosis observed in vitro.
\end{abstract}

\section{Keywords}

Apoptosis, Bisphenol A, Intra Uterine Growth Restriction, Placenta, Placental Vascularization

\#These authors equally contributed. 


\section{Introduction}

Bisphenol A (BPA) is an organic compound containing two phenolic groups and it is fundamental in the production of plastics, resins and polycarbonate. It can be found in food containers, baby bottles, plastics tableware and in epoxy resins used to produce films [1].

Some authors have defined BPA as an infamous molecule. BPA reaches the human organism through foods from plastic containers, especially if they are exposed to high temperatures and acidity [2].

Daily, a large number of people are exposed to BPA. This compound, as 17- $\beta$ estradiol, stimulates cellular responses and this role is more evident at exposure to low concentrations. BPA exposure is not only oral but also transdermal and inhalational [3]. BPA undergoes hepatic metabolism and is excreted in urine especially as glucuronide. Its phenolic structure allows it to interact with the estrogen receptor. For this reason, BPA is involved in many endocrine disorders such as infertility, early puberty, hormone dependent neoplasms and polycystic ovary syndrome [3].

Many studies have stressed the correlation between BPA and low birth weight (LBW) in both murine [4] [5] and human models [6] [7] [8]. Prolonged exposure has showed negative effects on embryo implantation [9], pregnancy establishment and continuation and differentiation of stromal cells into decidual ones [10].

Apart from liver, placenta also shows $\beta$-glucuronidase activity, which is responsible for BPA deconjugation [11]. For this reason, detectable levels of free BPA have been found in human placenta [12] [13]. Increased placental free BPA levels may represent a risk both for placental and for fetus development, due to BPA transfer across the feto-maternal barrier [14] [15].

Many studies describe BPA toxicity for reproduction and development both in rodents and in humans [16] [17] [18]; however, studies investigating placental effects of BPA are limited; few studies on mice have described some placental impairment during both early and late pregnancy. Subcutaneous BPA administration along the first seven days of pregnancy altered the development of decidua basalis and trophoblastic layers during implantation [5]. Oral administration of BPA affects the expression of the estrogen receptor $\beta(\operatorname{Er} \beta)$ and the progesterone receptor in late placenta [19]. Moreover, BPA exposed cytotrophoblast showed increased apoptosis, necrosis and the expression of the tumor necrosis factor $\alpha$ (TNF $\alpha$ ) [20]. Overall, evidence suggests that BPA may have adverse effects on placental tissue at environmentally relevant concentrations, with possible implications for embryo-fetal growth [4] [8] [21].

In humans, many studies report a decreased birth weight for babies born from mothers exposed to BPA [6] [8]. Low birth weight (LBW) is a condition characterized by a weight at birth less than 2500 grams. These children may be either preterm or term but small for gestational age (SGA). Assessed risk factors for LBW are: socioeconomic state, anemia, primiparity, low maternal height. However, 
birth weight reflects maternal health state and her nutrition state before conception and during pregnancy. Smoking and drug assumption during pregnancy are predisposing factors. In particular, smoke-related risk is dose-dependent. Also, maternal age is inversely correlated with LBW incidence; indeed, this phenomenon is more frequent among teen mothers [22].

LBW is an important predictor of infant mortality and it has a global incidence of $17 \%$. In terms of physiopathology, LBW is due to impaired placental circulation [23].

Though the relationship between BPA exposure and fetal weight outcome has been widely reported, the physio-pathological mechanisms are poorly understood. Placental angiogenesis has been reported as a possible target in the early phase of gestation. Tait et al. [24] demonstrated significant angiogenesis impairment in mice exposed to BPA early during pregnancy. Moreover, BPA has demonstrated to induce apoptosis in human cytotrophoblast cells exposed in vitro [20]. On the basis of these premises, the purpose of present study is to evaluate possible vascular alterations and apoptosis induction in placenta at a gestational terminal period on a rat model of oral exposure to low doses of BPA.

\section{Methods}

\subsection{Animals and BPA Exposure Protocol}

Six female $(200-250 \mathrm{~g})$ and three male $(250-300 \mathrm{~g})$ Wistar rats were used (Harlan Laboratories, San Pietro al Natisone (UD), Italy); they were housed under standard temperature and humidity conditions with a 12-h light-dark cycle (lights on from 07:00 a.m. to 07:00 p.m.) and with free access to standard fresh food and tap water. All the females had one pregnancy before the present experiment proving their fertility. Three male-female couples were randomly assorted and housed in the same cage until a vaginal plug was evident (day 0); the males were then coupled with the remaining females for another week. After the coupling period, each female was housed in a separate cage and was given BPA (3 rats) o vehicle (3 rats) in the drinking water. BPA was first dissolved in ethanol $(100 \mathrm{mg} / \mathrm{mL})$; this solution was then diluted 1:100 with ethanol; finally, 0.1 $\mathrm{mL}$ of the last solution were added to $1 \mathrm{~L}$ of tap water, resulting in a final concentration for BPA of $0.1 \mathrm{mg} / \mathrm{L}$. The vehicle consisted of ethanol $0.1 \mathrm{~mL} / \mathrm{L}$. The concentration of BPA was chosen to obtain a daily dose of $10 \mu \mathrm{g} / \mathrm{kg}$ bw, based on daily drinking consumption. This dose was lower than the limit for humans, currently considered "safe" by the European Food Safety Agency (ESFA) and the U.S. Environmental Protection Agency (EPA) [25] [26]. The pregnant females were then sacrificed by anesthetic overdose 15 days after day 0 ( 7 days before the expected delivery). All procedures fulfilled the requirements of the European Communities Council Directive of 22 September 2010 (2010/63EU). The research was approved by the local ethical committee of the University of Salerno and by the Ministry of Health of the Italian Government (aut. n. 45/2014-PR by the Ministry of Health). 


\subsection{Histology}

Rat placentas and embryos were fixed in $10 \%$ neutral buffered formalin and embedded in paraffin. Serial sections ( $4-\mu \mathrm{m}$ thick) were used for hematoxylin and eosin and immunohistochemistry. The presence and the number and area of clusters of glycogen cells were evaluated on two sections per placenta with PAS and PAS-diastase method.

\subsection{Immunohistochemical Staining and Analysis}

Four- $\mu \mathrm{m}$ sections were cut from the formalin-fixed paraffin-embedded tissue. We have evaluated for the study of micro-vessel density in the placental tissue and for apoptosis the following primary antibodies respectively: anti-von Willebrand factor (F-VIII-R-Ag polyclonal rabbit, Cell Marque, Rocklin, CA, USA, dilution 1:100), and anti-Caspase 9 (monoclonal mouse; Santa Cruz Biotechnology, Inc; no. sc-56076, dilution 1:100).

After antigen retrieval (microwave oven for 10 minutes at $250 \mathrm{~W}$ ) immunohistochemistry was carried out in a Benchmark automated staining system (Ventana Medical Systems, Tucson, AZ) according to the manufacturer's instructions and using Ventana reagents for the entire procedure.

Normal liver and adrenal gland tissue was chosen as positive control for F-VIII and Caspase 9.

The signal was further enhanced with the Ventana amplification kit. Slides were counterstained with hematoxylin, dehydrated, and mounted.

Two surgical pathologists performed a blinded evaluation of the slides for F-VIII and Caspase 9 without knowledge of BPA exposure data on a multi-headed microscope (Olympus BX43). For the evaluation, ten fields were examined for each section by each investigator. Micro-vessel density was assessed quantitatively by counting the number of vessels positive for F-VIII at 40 total magnifications for at least 10 fields.

In an attempt to characterize placental apoptosis, the expression levels for caspase 9 were determined in placental junctional and labyrinth zone: only the nuclear and cytoplasmic staining of trophoblastic cells were considered.

The intrapersonal and the interpersonal reproducibility were tested and the final data were merged.

\subsection{Statistical Analysis}

Data are expressed as means \pm standard deviation. Statistical analysis was performed using Statistica software (StatSoft, Oklahoma, USA) and Minitab (Minitab Inc, Pennsylvania, USA). After multiple attempts to normalize the data (e.g., natural $\log$, square root, inverse transformations), non-parametric statistical tests were employed (normality was tested via Shapiro-Wilk test). The comparison between groups was made with the Rank Sum Test, according to MannWhitney. The sample size was calculated using an expected effect size of 0.6 , an alpha value of 0.05 , and a beta value of 0.5 . This gave a minimum sample size of 
24 per group; expecting a minimum of 8 newborns for each female, three females were used for each group.

\section{Results}

\subsection{Maternal and Embryo Toxicity}

The number of live embryos was significantly higher in the BPA group (12.33 \pm 2.08 vs $10.00 \pm 3.46, \mathrm{p}<0.05)$. Placental diameter was significantly affected by the BPA treatment $(12.44 \pm 0.92 \mathrm{~mm}$ for the exposed rats, $13.10 \pm 1.09 \mathrm{~mm}$ for the non-exposed rats, $\mathrm{p}<0.05)$.

No significant effect was observed on embryo crown-rump and head major and minor axis lengths, total length and right forelimbs length. Longitudinal/transverse diameter ratio was significantly affected by BPA treatment $(2.57 \pm$ 0.29 for the exposed rats, $2.78 \pm 0.38$ for the non-exposed rats, $\mathrm{p}<0.05)$. All the results are reported in Table 1.

\subsection{Histological and Histo-Morphometrical Analysis}

The chorioallantoic placenta of the rat is composed of two morphologically distinct regions: junctional zone and labyrinth zone. The junctional zone or basal zone is located between uterine decidual tissue and the labyrinth zone. The labyrinth zone is located deep to the junctional zone and is in direct contact with the developing embryo.

An increase in degeneration and necrosis of giant cells, as well as the presence of larger and empty-appearing glycogen cells in the junctional zone were observed in BPA-exposed placentas. We also observed a significant glycogen decrease. BPA exposed placentas had larger and abnormal maternal vessels compared to controls. Maternal blood space areas were dilated and irregular in the BPA exposed group (Figure 1).

\subsection{Immunohistochemistry}

Micro-vessel count showed a lower vessel density in the placentas of BPA-exposed rats compared to controls (143.54 \pm 22.09 vs $124.86 \pm 19.15$ [vessels per field], $\mathrm{p}<0.001$ ) (Figure 2). No difference was observed between the two groups for Caspase- 9 expression $(54.91 \pm 19.72$ vs $52.26 \pm 16.82$ [vessels per field], $\mathrm{p}=0.56$ ) (Figure 3 ).

\section{Discussion}

In the present study, we report the effects on vascularization and apoptosis in placentas of rats chronically exposed to BPA. BPA exposure caused a decrease in terminal placental vascularization. This finding is consistent with the observation of the low vascularization observed by Tait et al. in the early placentation phase [24]. Nevertheless, apoptosis seems not to be affected by the chronic exposition to low doses of BPA. This finding, instead, seems to differ from the apoptosis induction by the in vitro BPA-exposure of human cytotrophoblasts 
Table 1. Fetuses and placental features.

\begin{tabular}{ccc}
\hline & BPA Group & Control Group \\
\hline Brood size & $12.33 \pm 2.08^{*}$ & $10.00 \pm 3.46$ \\
Fetal weight $(\mathrm{g})$ & $5.67 \pm 0.86$ & $5.40 \pm 1.98$ \\
Fetal length $(\mathrm{cm})$ & $3.44 \pm 0.41$ & $3.51 \pm 0.66$ \\
Fetal transversal length (mm) & $13.48 \pm 1.43$ & $13.45 \pm 1.38$ \\
Longitudinal/transverse diameter ratio & $2.57 \pm 0.29^{*}$ & $2.78 \pm 0.38$ \\
Placental diameter (mm) & $13.10 \pm 1.09^{*}$ & $12.44 \pm 0.92$ \\
Minor head axes (mm) & $10.25 \pm 0.86$ & $10.38 \pm 1.24$ \\
Major head axes (mm) & $15.79 \pm 0.98$ & $15.62 \pm 1.17$ \\
Right forelimbs length (mm) & $7.83 \pm 1.48$ & $7.93 \pm 1.21$ \\
Crown-rump length (mm) & $33.04 \pm 2.44$ & $33.76 \pm 2.18$ \\
Vessel (number per field) & $124.86 \pm 19.15^{*}$ & $143.54 \pm 22.09$ \\
Caspase-9 (number per field) & $54.91 \pm 19.72$ & $52.26 \pm 16.82$ \\
\hline
\end{tabular}

a. ${ }^{\star} \mathrm{p}<0.05$ compared to Control groups.
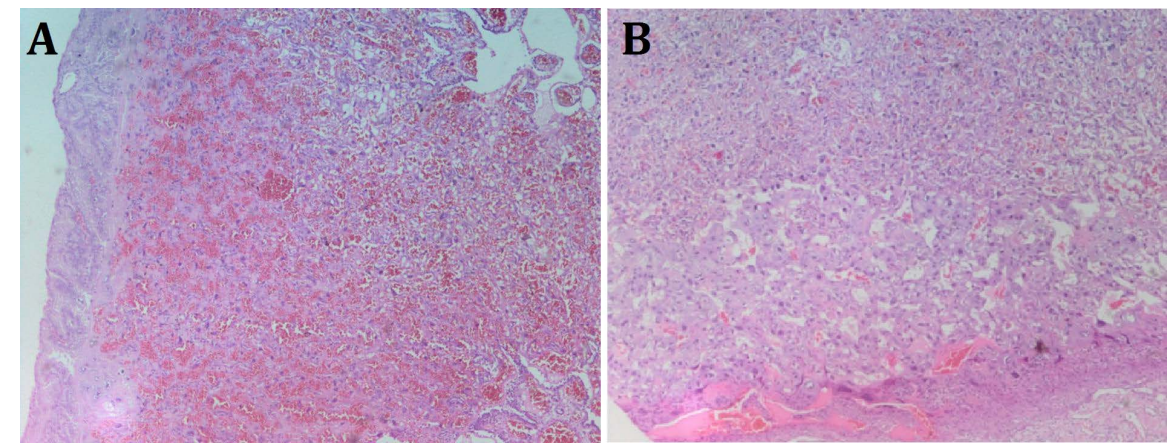

Figure 1. Placenta labyrinth layers of rat exposed to BPA (A) and to vehicle only (B). (Hematoxylin-eosin $\times 10$ ).
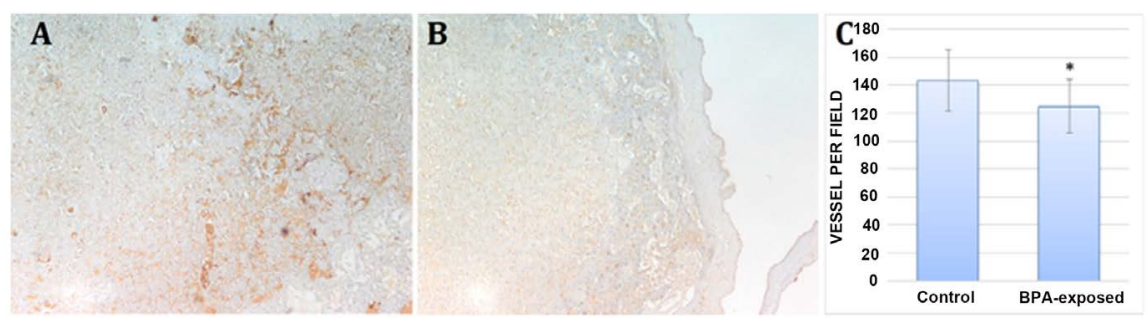

Figure 2. (A)F-VIII immunostaining of a non-exposed rat placenta (Immunoperoxydase $\times 10$ ). (B) BPA-exposed rat placenta showing greatly reduced micro-vessel density (Immunoperoxydase $\times 10$ ). (C) Means and standard deviation of vessels per field measured in controls and BPA-exposed rat. ${ }^{*}$ indicates a p-value $<0.05$.

reported by Benachour and Aris [20]. A greater number of embryos were also found in the BPA-treated group compared to the control group. This was an unexpected finding and could eventually be attributed to an estrogen-like effect of BPA on adult females. 

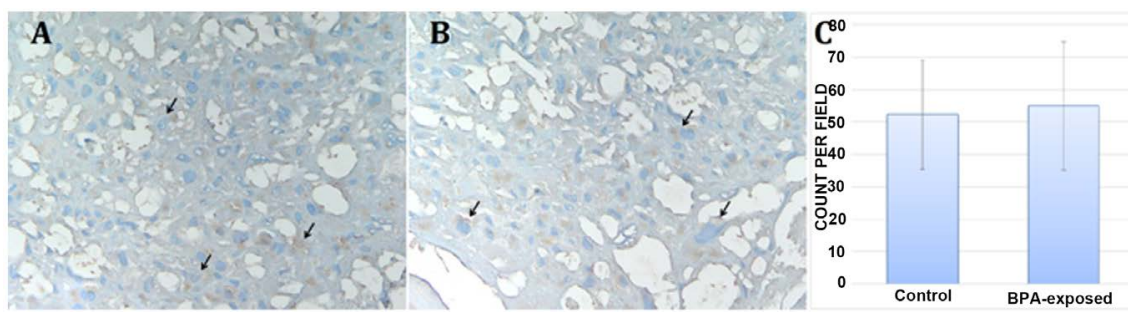

Figure 3. Immunohistochemistry of a non-exposed (A) and BPA-exposed (B) rat placenta showing cytoplasmic staining of trophoblastic cells for caspase 9 (Immunoperoxydase $\times 40$ ). (C) Means and standard deviation of counts per field measured in controls and BPA-exposed rat.

BPA is metabolized in placenta and it is transmitted vertically from mother to fetus as showed in murine and sheep models. Indeed, in the advanced phase of pregnancy BPA is conjugated and, as such, it is trapped in the fetal-placental unit. Conjugated BPA (BPA-G) is inactive. Sheep models showed that, during the early phase of pregnancy, the fetus has a low hepatic metabolism capacity. So, this may represent a critical moment for BPA exposure. Indeed, fetal hepatic microsomes have low expression of UGT2B1 and so a poor capacity to conjugate metabolites [27].

Later, the fetus acquires detoxificating capacity and can convert BPA in BPA-G. However, authors also showed that fetus metabolism can reactivate BPA from BPA-G, increasing toxic effects on fetal tissues [28]. Metabolite regeneration is possible thanks to the action of $\beta$-glucuronidase and phosphatases, expressed in many fetal tissues. Analysis on murine models showed that $4.4 \%$ of BPA is reconverted after its passage through placenta [27]. Through the deconjugation in fetal tissues, a considerable amount of active BPA accumulates in the fetal compartment. This process does not occur in early pregnancy, when the fetus has limited hepatic metabolic ability and placental detoxification has a protective role. So, BPA crosses placenta and reaches the fetus as a conjugated compound, then it is deconjugated in fetal tissues.

Five per cent of BPA infused to mothers is transmitted to fetus and pregnancy does not compromise toxic-kinetics of BPA. This is true for sheep models which have five or six layers separating maternal and fetal blood; in human placenta has only got three layers, so the transferred amount of BPA is supposed to be larger [29].

In murine uterine tissues exposed to $\mathrm{BPA}$, progesterone receptor (PGR) and its target gene HAND2 (heart and neural crest derivatives expressed 2) are markedly suppressed. This aspect is an important evidence for a negative role of BPA on pregnancy development because HAND2 rules embryo implantation [9]. These links are evident in human models as well. Analyses on maternal blood and amniotic fluid have suggested a correlation between BPA concentration and preterm birth or premature rupture of membranes. Actually, high BPA levels in maternal blood are more suggestive than the levels in the fetal compartment [30]. The same result appears dosing BPA in maternal urine at delivery 
[6], rather than in blood.

On this base, some authors have focused on changes in BPA concentration during pregnancy trimesters. They noticed that not global levels but levels in the last trimester are associated with preterm birth [31].

The relation between BPA exposure levels and birth weight decrease is dose-dependent [4].

BPA leads to miRNA alterations in placental cells and this suggests a new model of toxicity. Analyses on cytotrophoblastic cells show that some miRNAs are overexpressed, inhibiting proliferation and increasing susceptibility to DNA damage [32] [33]. Another BPA target is the TNF- $\alpha$ gene. Its increased expression is associated with a negative outcome of pregnancy and a higher risk of prematurity, preeclampsia, intrauterine growth retardation and miscarriage [20].

Studies in vitro on endometrial cells exposed to BPA during the decidualization process showed that this compound is responsible for a multi target dysregulation that prejudices pregnancy. Decidualization is a complex process which allows implantation. Decidualization of endometrial stromal cells (ESC) consists of morphologic and functional modifications under progesterone action during secretive phase. During this process, BPA interferes with estrogen and progesterone receptors and hCG/LH receptors. BPA reduces decidual prolactin expression but stimulates MIF (macrophage migration inhibitory factor) secretion, a cytokine produced by the endometrium having a role in implant, local inflammatory and immune response [10]. Probably, endometrial cells have a protective role on the fetus because they entrap BPA and reduce its placental availability. Indeed, while the direct exposure to BPA induces placental secretion of MIF and $\beta$-hCG, placental cultures previously exposed to endometrial stromal cells do not show this effect [34]. This hypothesis need of further investigation. Recently Turco et al. [35] have reported a 3D endometrial culture formation (organoids) able to show the in vitro endometrial function including the decidualization. Such a model can be used to deeply test this hypothesis.

Maybe the real target of BPA is placental angiogenesis. In particular, BPA is capable to inhibit placental vascularization, producing dilated and tortuous vessels as showed in murine models. Effects are dose dependent. Indeed, BPA50 (50 $\mathrm{mg} / \mathrm{kg}$ infusion) downregulates VEGFA (the leading regulator of placenta vasculogenesis and angiogenesis) and this gene alteration is responsible for morphogenetic modifications of junctional zone and placental vessels. BPA induces glycogen depletion and alters glucose homeostasis, increasing risk of gestational diabetes. Probably, placental morphological transformations create an imbalance in embryo nutrition and this is the basis of BPA negative effects on fetus growth [24].

BPA causes functional alterations on endometrial angiogenesis, as showed on endometrial endothelial cells (HEECs) in vitro. Authors demonstrated that endometrial cells act differently if they are in culture with stromal cells, rather than in monoculture [36]. When endometrial cells are in culture with stromal cells there are not BPA effects on proliferation. Morphological and functional altera- 
tions in endometrial vessels compromise placentation and implant. Moreover, HEECs are more susceptible than endothelial cells to endocrine disruptors as BPA. For example, HEECs have a high affinity to VEGF because they express a lot of VEGF receptors. BPA causes changes in mRNA expression of genes correlated to angiogenesis, especially for gene encoding VEGF-D protein. VEGF-D functions are to induce angiogenesis and lymph angiogenesis and to stimulate endothelial cells proliferation and migration. BPA effects are due to its action on androgen and glucocorticoid receptors, both expressed on endometrial endothelial and stromal cells [36].

A further demonstration of BPA negative effects on placentation is showed by studies on murine models exposed to BPA subcutaneously for the first eight days of pregnancy. Histological analysis showed poor development of trophoblastic giant cells that control maternal flow blood (and so placental angiogenesis), but also alteration of intervillous spaces and labyrinthine zones. It especially influences decidual basal and trophoblastic layer development. Moreover, BPA increases newborn number and their mortality. BPA stimulates stromal cells and causes hyperplasia of endometrial glands. Instead, the effect on trophoblast is indirect because trophoblast does not express estrogen receptors [5].

Several paths can be followed to monitor the apoptosis in cell cultures and in tissues. In this work we evaluated placental cell apoptosis by means of anti-caspase-9 antibody. Iida et al. [37] reported that BPA induces apoptosis in Sertoli cells via caspase activation. In particular, Teresaka et al. [38] report that this activation is mediated by caspase- 9 in HL-60 cells. In placenta, apoptosis plays a crucial role in its development during gestation. Several mechanisms of apoptosis were reported in normal placental tissue [39]. In particular, caspase-3 and caspase-8 (while not caspase-9) expression decrease were associated with preeclampsia-complicated pregnancies [39]. However, the role of caspase-9 is controversial, Afroza et al. [40] reported an increase of caspase-9 in placental tissue of women with a diagnosis of preeclampsia: so, caspase might have a role in the angiogenesis suppression and placental growth. However, our report suggests that BPA does not modify the placental expression of caspase-9, so the low micro-vessel formation in placentas of rats orally exposed to low dose of BPA would not be related to this mechanism.

Although the relationship between BPA exposition and LBW is widely reported [4] [8], our exposed fetuses do not differ from controls in term of weight. This phenomenon can be explained on the basis of two considerations: first it may depend from brood size, which can affect individual weight. Secondly, we interrupted the gestation after 15 days from vaginal plug, so the dams were sacrificed 7 days before the expected delivery, presumably fetal weights at term might have shown a significant difference.

\section{Conclusion}

Concluding in this study we report a decrease in placental micro-vessel amount in 
rats exposed to placental triggered by oral low doses of BPA. This phenomenon can lead to alterations in embryo nutrition and fetus growth, having a role in the pathogenesis of LBW. The apoptosis rate seems not to be affected.

\section{Acknowledgements}

This work has been partly supported by the fund PRIN-MIUR 2010-11 P.I. Rosaria Meccariello.

\section{Conflicts of Interest}

The authors declare no conflicts of interest regarding the publication of this paper.

\section{References}

[1] Chevalier, N. and Fenichel, P. (2015) Bisphenol A: Targeting Metabolic Tissues. Reviews in Endocrine and Metabolic Disorders, 16, 299-309. https://doi.org/10.1007/s11154-016-9333-8

[2] Lazurova, Z. and Lazurova, I. (2013) The Environmental Estrogen Bisphenol A and Its Effects on the Human Organism. Vnitrnní lékařství, 59, 466-471.

[3] Konieczna, A., Rutkowska, A. and Rachon, D. (2015) Health Risk of Exposure to Bisphenol A (BPA). Roczniki Państwowego Zakładu Higieny, 66, 5-11.

[4] Miao, M., Yuan, W., Zhu, G., He, X. and Li, D.-K. (2011) In Utero Exposure to Bisphenol-A and Its Effect on Birth Weight of Offspring. Reproductive Toxicology, 32, 64-68. https://doi.org/10.1016/j.reprotox.2011.03.002

[5] Tachibana, T., et al. (2007) Effects of Bisphenol A (BPA) on Placentation and Survival of the Neonates in Mice. Journal of Reproduction and Development, 53, 509-514.

[6] Huo, W., et al. (2015) Maternal Urinary Bisphenol A Levels and Infant Low Birth Weight: A Nested Case-Control Study of the Health Baby Cohort in China. Environment International, 85, 96-103. https://doi.org/10.1016/j.envint.2015.09.005

[7] Troisi, J., et al. (2014) Placental Concentrations of Bisphenol A and Birth Weight from Births in the Southeastern U.S. Placenta, 35, 947-952. https://doi.org/10.1016/j.placenta.2014.08.091

[8] Snijder, C.A., et al. (2013) Fetal Growth and Prenatal Exposure to Bisphenol A: The Generation R Study. Environmental Health Perspectives, 121, 393-398. https://doi.org/10.1289/ehp.1205296

[9] Li, Q., Davila, J., Kannan, A., Flaws, J.A., Bagchi, M.K. and Bagchi, I.C. (2016) Chronic Exposure to Bisphenol A Affects Uterine Function during Early Pregnancy in Mice. Endocrinology, 157, 1764-1774. https://doi.org/10.1210/en.2015-2031

[10] Mannelli, C., et al. (2015) Bisphenol A Modulates Receptivity and Secretory Function of Human Decidual Cells: An in Vitro Study. Reproduction, 150, 115-125. https://doi.org/10.1530/REP-14-0601

[11] Ginsberg, G. and Rice, D.C. (2009) Does Rapid Metabolism Ensure Negligible Risk from Bisphenol A? Environmental Health Perspectives, 117, 1639-1643. https://doi.org/10.1289/ehp.0901010

[12] Cao, X.-L., Zhang, J., Goodyer, C.G., Hayward, S., Cooke, G.M. and Curran, I.H.A. (2012) Bisphenol A in Human Placental and Fetal Liver Tissues Collected from 
Greater Montreal Area (Quebec) during 1998-2008. Chemosphere, 89, 505-511. https://doi.org/10.1016/j.chemosphere.2012.05.003

[13] Vandenberg, L.N., Hauser, R., Marcus, M., Olea, N. and Welshons, W.V. (2007) Human Exposure to Bisphenol A (BPA). Reproductive Toxicology, 24, 139-177.

[14] Corbel, T., et al. (2014) Bidirectional Placental Transfer of Bisphenol A and Its Main Metabolite, Bisphenol A-Glucuronide, in the Isolated Perfused Human Placenta. Reproductive Toxicology, 47, 51-58. https://doi.org/10.1016/j.reprotox.2014.06.001

[15] Morck, T.J., et al. (2010) Placental Transport and in Vitro Effects of Bisphenol A. Reproductive Toxicology, 30, 131-137. https://doi.org/10.1016/j.reprotox.2010.02.007

[16] Richter, C.A., et al. (2007) In Vivo Effects of Bisphenol A in Laboratory Rodent Studies. Reproductive Toxicology, 24, 199-224. https://doi.org/10.1016/j.reprotox.2007.06.004

[17] Rubin, B.S. (2011) Bisphenol A: An Endocrine Disruptor with Widespread Exposure and Multiple Effects. The Journal of Steroid Biochemistry and Molecular Biology, 127, 27-34. https://doi.org/10.1016/j.jsbmb.2011.05.002

[18] Guida, M., et al. (2015) Bisphenol A and Congenital Developmental Defects in Humans. Mutation Research, 774, 33-39. https://doi.org/10.1016/j.mrfmmm.2015.02.007

[19] Imanishi, S., et al. (2003) Effects of Oral Exposure of Bisphenol A on mRNA Expression of Nuclear Receptors in Murine Placentae Assessed by DNA Microarray. Journal of Reproduction and Development, 49, 329-336. https://doi.org/10.1262/jrd.49.329

[20] Benachour, N. and Aris, A. (2009) Toxic Effects of Low Doses of Bisphenol-A on Human Placental Cells. Toxicology and Applied Pharmacology, 241, 322-328. https://doi.org/10.1016/j.taap.2009.09.005

[21] Chou, W.-C., Chen, J.-L., Lin, C.-F., Chen, Y.-C., Shih, F.-C. and Chuang, C.-Y. (2011) Biomonitoring of Bisphenol A Concentrations in Maternal and Umbilical Cord Blood in Regard to Birth Outcomes and Adipokine Expression: A Birth Cohort Study in Taiwan. Environmental Health, 10, 94. https://doi.org/10.1186/1476-069X-10-94

[22] Khan, A., Nasrullah, F.D. and Jaleel, R. (2016) Frequency and Risk Factors of Low Birth Weight in Term Pregnancy. Pakistan Journal of Medical Sciences, 32, 138-142.

[23] Valero De Bernabe, J., et al. (2004) Risk Factors for Low Birth Weight: A Review. European Journal of Obstetrics \& Gynecology and Reproductive Biology, 116, 3-15. https://doi.org/10.1016/j.ejogrb.2004.03.007

[24] Tait, S., Tassinari, R., Maranghi, F. and Mantovani, A. (2015) Bisphenol A Affects Placental Layers Morphology and Angiogenesis during Early Pregnancy Phase in Mice. Journal of Applied Toxicology, 35, 1278-1291. https://doi.org/10.1002/jat.3176

[25] E. F. S. Authority (EFSA), et al. (2017) Bisphenol A (BPA) Hazard Assessment Protocol. EFSA Supporting Publications, 1354E.

[26] USEPA (2010) Bisphenol A Action Plan. United States Environmental Protection Agency, Washington DC.

[27] Nishikawa, M., Iwano, H., Yanagisawa, R., Koike, N., Inoue, H. and Yokota, H. (2010) Placental Transfer of Conjugated Bisphenol A and Subsequent Reactivation in the Rat Fetus. Environmental Health Perspectives, 118, 1196-1203.

https://doi.org/10.1289/ehp.0901575 
[28] Corbel, T., et al. (2015) Conjugation and Deconjugation Reactions within the Fetoplacental Compartment in a Sheep Model: A Key Factor Determining Bisphenol A Fetal Exposure. Drug Metabolism \& Disposition, 43, 467-476. https://doi.org/10.1124/dmd.114.061291

[29] Corbel, T., et al. (2013) Bisphenol A Disposition in the Sheep Maternal-Placental-Fetal Unit: Mechanisms Determining Fetal Internal Exposure. Biology of Reproduction, 89, 11. https://doi.org/10.1095/biolreprod.112.106369

[30] Behnia, F., Peltier, M., Getahun, D., Watson, C., Saade, G. and Menon, R. (2016) High Bisphenol A (BPA) Concentration in the Maternal, But Not Fetal, Compartment Increases the Risk of Spontaneous Preterm Delivery. The Journal of Maternal-Fetal \& Neonatal Medicine, 29, 3583-3589. https://doi.org/10.3109/14767058.2016.1139570

[31] Cantonwine, D.E., Ferguson, K.K., Mukherjee, B., McElrath, T.F. and Meeker, J.D. (2015) Urinary Bisphenol A Levels during Pregnancy and Risk of Preterm Birth. Environmental Health Perspectives, 123, 895-901. https://doi.org/10.1289/ehp.1408126

[32] Avissar-Whiting, M., et al. (2010) Bisphenol A Exposure Leads to Specific Microrna Alterations in Placental Cells. Reproductive Toxicology, 29, 401-406. https://doi.org/10.1016/j.reprotox.2010.04.004

[33] De Felice, B., et al. (2015) Genome-Wide microRNA Expression Profiling in Placentas from Pregnant Women Exposed to BPA. BMC Medical Genomics, 8, 56. https://doi.org/10.1186/s12920-015-0131-z

[34] Mannelli, C., et al. (2014) Bisphenol A Alters Beta-hCG and MIF Release by Human Placenta: An in Vitro Study to Understand the Role of Endometrial Cells. Mediators of Inflammation, 2014, Article ID: 635364. https://doi.org/10.1155/2014/635364

[35] Turco, M.Y., et al. (2017) Long-Term, Hormone-Responsive Organoid Cultures of Human Endometrium in a Chemically Defined Medium. Nature Cell Biology, 19, 568. https://doi.org/10.1038/ncb3516

[36] Helmestam, M., Davey, E., Stavreus-Evers, A. and Olovsson, M. (2014) Bisphenol A Affects Human Endometrial Endothelial Cell Angiogenic Activity in Vitro. Reproductive Toxicology, 46, 69-76. https://doi.org/10.1016/j.reprotox.2014.03.002

[37] Iida, H., Maehara, K., Doiguchi, M., Mōri, T. and Yamada, F. (2003) Bisphenol A Induced Apoptosis of Cultured Rat Sertoli Cells. Reproductive Toxicology, 17, 457-464. https://doi.org/10.1016/S0890-6238(03)00034-0

[38] Terasaka, H., Kadoma, Y., Sakagami, H. and Fujisawa, S. (2005) Cytotoxicity and Apoptosis-Inducing Activity of Bisphenol A and Hydroquinone in. Anticancer Research, 25, 2241-2247.

[39] Sokolov, D.I., et al. (2009) Regulatory Mechanisms for Apoptosis in Placental Tissue during Normal Pregnancy and Gestosis-Complicated Pregnancy. Bulletin of Experimental Biology and Medicine, 148, 766-770. https://doi.org/10.1007/s10517-010-0812-8

[40] Afroze, S.H., et al. (2016) Apoptotic and Stress Signaling Markers Are Augmented in Preeclamptic Placenta and Umbilical Cord. BBA Clinical, 6, 25-30.

https://doi.org/10.1016/j.bbacli.2016.05.003 